\title{
Relationship between pneumonitis induced by immune checkpoint inhibitors and the underlying parenchymal status: a retrospective study
}

\author{
Chiara Pozzessere ${ }^{1,7}$, Hasna Bouchaab ${ }^{2,7}$, Raphael Jumeau ${ }^{3}$, Igor Letovanec ${ }^{4}$ \\ Cécile Daccord $\mathbb{0}^{5}$, Jean Bourhis ${ }^{3}$, John O. Prior (10) ${ }^{1}$, Solange Peters ${ }^{2}$, \\ Romain Lazor (1) ${ }^{5,8}$ and Catherine Beigelman-Aubry ${ }^{6,8}$
}

Affiliations: ${ }^{1}$ Dept of Nuclear Medicine and Molecular Imaging, Lausanne University Hospital and University of Lausanne, Lausanne, Switzerland. ${ }^{2}$ Medical Oncology Dept, Lausanne University Hospital and University of Lausanne, Lausanne, Switzerland. ${ }^{3}$ Dept of Radiation Oncology, Lausanne University Hospital and University of Lausanne, Lausanne, Switzerland. Institute of Pathology, Lausanne University Hospital and University of Lausanne, Lausanne, Switzerland. ${ }^{5}$ Respiratory Medicine Dept, Lausanne University Hospital and University of Lausanne, Lausanne, Switzerland. ${ }^{6}$ Dept of Radiodiagnostic and Interventional Radiology, Lausanne University Hospital and University of Lausanne, Lausanne, Switzerland. ${ }^{7}$ Joint first authors. ${ }^{8}$ Joint senior authors.

Correspondence: Romain Lazor, Service de Pneumologie, Centre Hospitalier Universitaire Vaudois, BU44.07.2137, Rue du Bugnon 44, CH-1011 Lausanne, Switzerland. E-mail: romain.lazor@achuv.ch

ABSTRACT In patients with primary or secondary lung tumour treated with immune checkpoint inhibitors, immune-related pneumonitis is a rare adverse event but may evolve to respiratory failure. Prompt management is required and usually consists of treatment interruption and immunosuppressive drug administration. The aim of this study was to evaluate relationships between immune-related pneumonitis and pre-existing parenchymal status, especially tumour location and history of chest radiotherapy.

Computed tomography (CT) scans of patients with immune-related pneumonitis were retrospectively reviewed. Pattern, distribution and extent of pneumonitis were assessed in six lung regions. In patients who received radiotherapy, the extent of pneumonitis was evaluated according to the radiation field.

Among 253 patients treated with immunotherapy, 15 cases of immune-related pneumonitis were identified. 10 had previous or concomitant chest radiotherapy in addition to immunotherapy. At CT scan, 29 (33\%) out of 88 regions encompassed the primary tumour $(n=4)$, a lung metastasis $(n=4)$ and/or radiation fields $(n=21)$. A significantly higher prevalence of parenchymal involvement by immune-related pneumonitis occurred within areas of primary or metastatic malignancy and/or radiation field $(97 \%)$ as compared to other areas $(3 \%$, $\mathrm{p}=0.009$ ). Lung regions affected by the primary tumour, metastasis or radiotherapy had a higher probability of immune-related pneumonitis than others ( $\mathrm{OR} 10.8, \mathrm{p}=0.024)$. An organising pneumonia $(\mathrm{OP})$ pattern was more frequent after radiotherapy $(70 \%$ versus $0 \%, \mathrm{p}=0.024)$, whereas nonspecific interstitial pneumonia features were more commonly seen in radiotherapy-naive patients ( $100 \%$ versus $10 \%, \mathrm{p}=0.002)$.

In patients with primary or secondary lung tumour treated with immune checkpoint inhibitors, immunerelated pneumonitis is preferentially located within lung areas involved by tumour and/or radiation fields.

@ERSpublications

In patients with primary or secondary lung tumour treated by immune checkpoint inhibitors, immune-related pneumonitis induced by these agents is preferentially located within lung areas involved by tumour and/or radiation fields http://bit.ly/2NJZmGx

Cite this article as: Pozzessere C, Bouchaab H, Jumeau R, et al. Relationship between pneumonitis induced by immune checkpoint inhibitors and the underlying parenchymal status: a retrospective study. ERJ Open Res 2020; 6: 00165-2019 [https://doi.org/10.1183/23120541.00165-2019]. 


\section{Introduction}

Immune checkpoint inhibitors (ICIs) represent an emerging class of anticancer therapy, which has modified the outcome of several metastatic solid tumours, as well as locally advanced melanoma and non-small cell lung cancer. ICIs are antagonist antibodies that target immune checkpoints located either on T-cells, such as PD-1 and cytotoxic T-lymphocyte antigen-4, or on neoplastic cells, such as PD-1 ligand. These checkpoints are physiologically important for immune homeostasis and activated by cancer cells to evade immune system destruction [1]. ICIs interfere with this process, and reactivate the priming and effector phases of the immune response against the neoplastic cells, which has been shown to result in a systemic, complete and durable cancer response in a subset of patients. Recently, a synergistic effect of immunotherapy and radiotherapy (RT) has been suggested in selected patients, with several studies demonstrating a significant prolonged progression-free survival and overall survival when immunotherapy is combined with previous or concomitant RT [2-9].

Although ICIs have an overall safe profile, alone or in combination with RT, specific complications called immune-related adverse events (irAEs) may occur in up to $85 \%$ of patients [10]. Fortunately, most of them are manageable grade 1 or 2 events. Among them, immune-related pneumonitis (IP) is rare, occurring in around $2-5 \%$ of patients ( $1 \%$ grade $3-5$ ), but is relevant because it may lead to respiratory failure [11]. IP generally responds to immunotherapy interruption and steroids or immunosuppressive drugs, but it requires a prompt and correct identification [10], and may impact the subsequent therapeutic management.

An early and accurate diagnosis of IP may be challenging since the clinical and imaging features are not specific. Several imaging patterns have been reported such as organising pneumonia, nonspecific interstitial pneumonia (NSIP), hypersensitivity pneumonitis, acute interstitial pneumonitis/diffuse alveolar damage syndrome (DAD) and in some cases, no specific appearance (not otherwise specified pneumonia) $[12,13]$. Moreover, neither specific triggers nor underlying clinical or pathological lung conditions have been correlated with IP occurrence. Recently, the development of IP in previously irradiated areas of the lung has been reported in a few cases, suggesting a "hyperactivation" of T-cells in the RT field or a "radiation recall" effect [14-16]. However, to date, insufficient data are available to confirm this hypothesis. The aim of this retrospective study was to evaluate the relationships between IP and underlying lung conditions, especially tumour location and previous RT.

\section{Materials and methods}

\section{Patients}

This retrospective observational study was performed after obtaining institutional research informed consent from all patients, allowing anonymous data analysis. All patients with a suspicion of IP discussed in our multidisciplinary board were reviewed in this retrospective case series. All patients received ICIs for a neoplastic disease within clinical trials or according to routine care (RC) at our institution. All biological and radiological examinations were performed according to RC.

Inclusion criteria were the following: 1) New onset of a clinical or radiological event suspicious for IP during ICI treatment: terminology and severity of the adverse event were assessed using the Common Terminology Criteria for Adverse Events (CTCAE) version 4.0. 2) Chest computed tomography (CT) scan. 3) Bronchoalveolar lavage (BAL) or biopsy performed at the time of IP. When BAL or biopsy were not performed because of IP severity, improvement after ICI interruption and steroids was considered as a diagnostic confirmation of IP. Cases with sarcoidosis-like irAEs or lung infection were excluded.

\section{Imaging assessment}

Three independent readers retrospectively reviewed the chest CT scans: two experienced thoracic oncology radiologists (C. Pozzessere and C. Beigelman-Aubry) and one respiratory physician specialised in interstitial lung diseases (R. Lazor). The readers analysed imaging acquired at the following time points: before immunotherapy, before the onset of IP, and at IP diagnosis. For the purpose of this analysis, the last CT scan before appearance of IP was considered as a "baseline scan" and the one at the time of IP diagnosis as the "IP scan". In patients who underwent RT, CT scans before and after RT were also assessed, and the presence or absence of RT-induced pneumonia was recorded. Pre-existing radiation-induced abnormalities were not considered as immune-related in the assessment of IP features.

Each lung was divided into three regions: the upper lung from the apex to the carina, the middle lung from the carina to the lower pulmonary veins and the lower lung from the lower pulmonary veins to the diaphragm, making a total of six lung regions per patient. The specific pulmonary findings and their distribution were assessed according to the Fleischner Society terminology [17] and the extent of involvement for each region was determined on a five-point Likert scale $(0 \%, 1-25 \%, 26-50 \%, 51-75 \%$ 
and $76 \%-100 \%)$. Then, an imaging pattern was attributed according to the American Thoracic Society/ European Respiratory Society (ATS/ERS) classification of idiopathic interstitial pneumonias [18].

For patients who received RT, all treatments were delivered with intensity modulated RT techniques using helical tomotherapy or volumetric modulated arc therapy. RT plans were coregistered with the baseline scan and the IP scan to evaluate whether IP-related lesions were within the RT field. In addition, the percentage of IP within and outside the RT field was visually assessed, ranging from $100 \%$ when the involvement was entirely confined within the RT field to $0 \%$ when no abnormalities were detected in the RT field. A 5-Gy isodose line was chosen to determine the edge of RT fields.

CT studies after ICI interruption and steroids administration were also reviewed to assess the resolution of IP abnormalities.

\section{Cytological and histological analysis}

Cytological and histological specimens were analysed by two lung pathologists. The BAL differential cell count was used in combination with imaging to classify each case into clinical patterns according to the ATS/ERS guidelines [18]. Infection was ruled out by BAL microbiology analysis and/or clinical course.

\section{Statistical analysis}

The Fischer's exact test was used to evaluate the prevalence of IP in lung regions with or without previous tumour, metastasis or RT, and the differences in CT pattern between RT-treated and RT-naïve patients. The proportion of lung parenchyma infiltration by IP was measured on CT and dichotomised using a threshold $\geqslant 1 \%$ versus $0 \%$. The probability of IP by lung regions was analysed by logistic regression according to the presence or absence of previous tumour, metastasis or RT. Statistical analyses were conducted on Stata 15.1 (StataCorp, College Station, TX, USA) with $\mathrm{p}<0.05$ as significance level.

\section{Results}

Among 253 cancer patients treated with ICIs at our institution, 15 patients (10 males; median age 68 years, range $48-88$ years) presenting with symptoms of IP according to CTCAE version 4.0 criteria $(n=9)$ or compatible radiological findings $(n=6)$ were retrospectively included. Four of them were included in clinical trials and 11 treated according to RC. Eight other patients were excluded because of sarcoid-like reactions $(n=5)$ or lung infection $(n=3)$. The diagnosis of IP was confirmed by BAL $(n=11)$ and/or tissue sampling $(n=2)$. In two patients, recovery after immunotherapy interruption and steroid administration was considered as a diagnostic confirmation of IP.

Nine patients had lung cancer (61\%), four had melanoma (27\%), one had a parotid epidermoid carcinoma (6\%) and one had oesophageal cancer (6\%). Six patients $(40 \%)$ were treated with nivolumab, two (13\%) with ipilimumab, four (27\%) with combined ipilimumab and nivolumab (followed by nivolumab maintenance therapy in three), one (6\%) with durvalumab and tremelimumab, and two (13\%) with pembrolizumab. Patient characteristics are shown in tables 1 and 2.

10 patients $(67 \%)$ underwent RT, six of whom did so before ICIs, while three patients received concurrent ICIs and RT (20\%), and one (case 1) had RT both before and during ICIs. Seven patients developed imaging features compatible with radiation-induced pneumonitis within 6 months after RT. RT treatments are detailed in table 3.

During the 18 months prior to IP, four patients (27\%) had a pulmonary infection (one Pseudomonas aeruginosa pneumonia, one rhinovirus bronchiolitis, one Pneumocystis jiroveci pneumonia, one community-acquired pneumonia), one (6\%) had chemotherapy-induced pneumonia (case 6) and one (6\%) underwent cryoablation of a lung metastasis (case 2). Other lung disorders included chronic obstructive pulmonary disease $(n=6)$.

According to CTCAE version 4, the severity of IP was classified as grade 1 in six cases (40\%), grade 3 in seven cases (47\%) and grade 4 in two cases (13\%). In grade 1 patients, IP was initially identified by scheduled imaging for restaging (positron emission tomography/CT in three and CT in three). BAL was performed in 11 patients (74\%), whereas the diagnosis of IP was histologically proven in two, including one by transbronchial biopsy and one with a surgical specimen (13\%). In two other patients (13\%), the severity of the clinical condition did not allow any invasive procedures and diagnosis was made through improvement under immunosuppressive treatment. BAL results are detailed in table 4. A predominantly lymphocytic or mixed pattern was observed in all but one patient with respiratory failure, in whom BAL demonstrated a neutrophilic profile.

Imaging characteristics are shown in tables 4 and 5. On the baseline scan, RT-naïve tumoral lesions related to primary lung tumour were seen in four regions and metastasis in four regions (table 5). 13 RT-treated neoplastic lesions - either primary lung cancer or metastasis - resulted in 21 irradiated regions on the 


\begin{tabular}{|c|c|c|c|c|c|c|c|c|c|c|c|c|}
\hline $\begin{array}{l}\text { Case } \\
\text { number }\end{array}$ & $\begin{array}{l}\text { Age } \\
\text { years }\end{array}$ & Sex & $\begin{array}{l}\text { Time of } \\
\text { diagnosis }\end{array}$ & Tumour histology & Stage & $\begin{array}{l}\text { Intrathoracic tumour } \\
\text { manifestations at } \\
\text { diagnosis }\end{array}$ & Chemotherapy & RT & $\begin{array}{l}\text { Intrathoracic tumour } \\
\text { manifestations before RT }\end{array}$ & Time of RT & $\begin{array}{l}\text { Events other than RT in the } \\
18 \text { months before the onset } \\
\text { of IP }\end{array}$ & $\begin{array}{l}\text { Intrathoracic tumour } \\
\text { manifestations before ICI }\end{array}$ \\
\hline 1 & 88 & M & May 2014 & $\begin{array}{l}\text { Metastatic squamous cell } \\
\text { carcinoma of the head } \\
\text { and neck }\end{array}$ & IV & $\begin{array}{l}\text { Bilateral lung metastasis, } \\
\text { right hilar metastasis }\end{array}$ & NA & Yes & $\begin{array}{l}\text { Bilateral lung metastasis, right } \\
\text { hilar metastasis }\end{array}$ & $\begin{array}{l}\text { Dec } 2014 \text { to Feb } \\
2016\end{array}$ & $\begin{array}{l}\text { Pseudomonas aeruginosa } \\
\text { pneumonia }\end{array}$ & $\begin{array}{l}\text { Bilateral lung metastasis, right } \\
\text { hilar metastasis }\end{array}$ \\
\hline 2 & 70 & M & Jan 2013 & $\begin{array}{l}\text { 1) Mixed small cell } \\
\text { carcinoma and } \\
\text { squamous cell } \\
\text { carcinoma of the lung } \\
\text { 2) Squamous cell } \\
\text { carcinoma of the lung }\end{array}$ & $\mathrm{IIB}$ & $\begin{array}{l}\text { Primary carcinoma (mass) } \\
\text { LUL (1), synchronous } \\
\text { primary carcinoma RUL } \\
\text { (2), left-sided metastatic } \\
\text { mediastinal and hilar } \\
\text { lymph nodes, small left } \\
\text { pleural effusion }\end{array}$ & Cisplatin/etoposide & Yes & $\begin{array}{l}\text { Primary carcinoma (mass) LUL } \\
\text { (1), synchronous primary } \\
\text { carcinoma RUL (2), } \\
\text { left-sided metastatic } \\
\text { mediastinal and hilar lymph } \\
\text { nodes, small left pleural } \\
\text { effusion }\end{array}$ & Jan to July 2013 & Lung metastasis cryoablation & $\begin{array}{l}\text { LLL metastasis extending to } \\
\text { the chest wall }\end{array}$ \\
\hline 3 & 69 & M & May 2016 & $\begin{array}{l}\text { Squamous cell carcinoma } \\
\text { of the lung }\end{array}$ & IV & $\begin{array}{l}\text { Primary carcinoma of the } \\
\text { RUL, homolateral LN } \\
\text { metastasis, thoracic spine } \\
\text { metastasis T7-T11 }\end{array}$ & No & Yes & $\begin{array}{l}\text { Primary carcinoma of the RUL, } \\
\text { homolateral LN metastasis, } \\
\text { thoracic spine metastasis } \\
\text { T7-T11 }\end{array}$ & June 2016 (spine) & No & $\begin{array}{l}\text { Primary carcinoma of the RUL, } \\
\text { homolateral LN metastasis, } \\
\text { thoracic spine metastasis } \\
\text { T7-T11 }\end{array}$ \\
\hline 4 & 58 & M & Jan 2010 & Melanoma & IB & None & No & Yes & $\begin{array}{l}\text { Lung metastasis RLL lafter } \\
\text { incomplete surgical } \\
\text { resection) }\end{array}$ & July 2015 & $\begin{array}{l}\text { Community-acquired } \\
\text { pneumonia }\end{array}$ & New nodule RUL \\
\hline 5 & 68 & $\mathrm{~F}$ & June 2015 & Melanoma & IIIB & $\begin{array}{l}\text { Nonhypermetabolic nodule } \\
\text { LLL, chest wall metastasis }\end{array}$ & No & No & NA & No & $\begin{array}{l}\text { Rhinovirus bronchiolitis, } \\
\text { recurrence of chest wall and } \\
\text { liver metastasis }\end{array}$ & Chest wall metastasis \\
\hline 6 & 80 & M & June 2016 & $\begin{array}{l}\text { Squamous cell carcinoma } \\
\text { of the lung }\end{array}$ & IV & $\begin{array}{l}\text { Primary carcinoma LLL, } \\
\text { previous left upper } \\
\text { lobectomy in } 2014 \text { for } \\
\text { adenocarcinoma of the } \\
\text { LUL }\end{array}$ & $\begin{array}{l}\text { Carboplatin/ } \\
\text { vinorelbine (first } \\
\text { carcinomal then } \\
\text { carboplatin/ } \\
\text { gemcitabine }\end{array}$ & No & NA & No & Drug-induced thrombopenia & $\begin{array}{l}\text { Primary carcinoma LLL, } \\
\text { previous left upper } \\
\text { lobectomy }\end{array}$ \\
\hline 7 & 75 & M & March 2016 & Lung adenocarcinoma & IV & Primary carcinoma LUL & No & No & NA & No & No & Primary carcinoma LUL \\
\hline 8 & 59 & $\mathrm{~F}$ & July 2015 & $\begin{array}{l}\text { Squamous cell carcinoma } \\
\text { of the lung }\end{array}$ & IV & $\begin{array}{l}\text { Primary carcinoma (mass) } \\
\text { RUL }\end{array}$ & $\begin{array}{l}\text { Cisplatin/ } \\
\text { vinorelbine then } \\
\text { carboplatin/ } \\
\text { vinorelbine }\end{array}$ & Yes & Surgical resection of RUL & Jan to Feb 2016 & $\begin{array}{l}\text { Pneumocystis jiroveci } \\
\text { pneumonia }\end{array}$ & $\begin{array}{l}\text { Local recurrence LLL } \\
\text { metastasis }\end{array}$ \\
\hline 9 & 51 & $\mathrm{~F}$ & Jan 2014 & Small cell lung cancer & IB & $\begin{array}{l}\text { Primary carcinoma (mass) } \\
\text { RLL }\end{array}$ & Cisplatin/etoposide & Yes & Primary carcinoma (mass) RLL & $\begin{array}{l}\text { Feb } 2015 \text { to } \\
\text { March } 2016\end{array}$ & No & Primary carcinoma (mass) RLL \\
\hline 10 & 57 & $\mathrm{~F}$ & Feb 2015 & Lung adenocarcinoma & IV & $\begin{array}{l}\text { Primary carcinoma (mass) } \\
\text { LUL }\end{array}$ & $\begin{array}{l}\text { Pemetrexed then } \\
\text { carboplatin/ } \\
\text { pemetrexed }\end{array}$ & Yes & Primary carcinoma (mass) LUL & Sept to Oct 2015 & No & Primary carcinoma (mass) LUL \\
\hline 11 & 48 & M & March 2007 & Right arm melanoma & IB & No & No & No & NA & No & No & $\begin{array}{l}\text { Melanoma metastasis (mass) } \\
\text { RUL }\end{array}$ \\
\hline 12 & 71 & M & Feb 2017 & $\begin{array}{l}\text { Oesophageal } \\
\text { adenocarcinoma }\end{array}$ & IV & $\begin{array}{l}\text { Oesophageal carcinoma, } \\
\text { locoregional metastatic } \\
\text { lymph nodes }\end{array}$ & No & Yes & Chest wall metastasis & March 2017 & No & Chest wall metastasis \\
\hline 13 & 88 & M & Oct 2015 & $\begin{array}{l}\text { Squamous cell carcinoma } \\
\text { of the lung }\end{array}$ & IIIA & $\begin{array}{l}\text { Primary carcinoma of RUL } \\
\text { extending to chest wall, } \\
\text { mediastinal and right hilar } \\
\text { lymph node metastasis }\end{array}$ & Gemcitabine & Yes & $\begin{array}{l}\text { Primary carcinoma of RUL } \\
\text { extending to chest wall, } \\
\text { mediastinal and right hilar } \\
\text { lymph node metastasis }\end{array}$ & $\begin{array}{l}\text { Nov } 2015 \text { to Feb } \\
2016\end{array}$ & No & $\begin{array}{l}\text { Primary carcinoma of RUL } \\
\text { extending to chest wall, } \\
\text { mediastinal and right hilar } \\
\text { lymph node metastasis }\end{array}$ \\
\hline 14 & 62 & M & Feb 2007 & Melanoma & IV & $\begin{array}{l}\text { Mediastinal and hilar } \\
\text { metastasis and metastasis } \\
\text { of the RLL }\end{array}$ & Dacarbazine & No & $\mathrm{NA}$ & No & No & $\begin{array}{l}\text { Mediastinal and hilar } \\
\text { metastasis and metastasis } \\
\text { of the RLL }\end{array}$ \\
\hline 15 & 72 & M & Oct 2012 & Lung adenocarcinoma & $11 \mathrm{~b}$ & $\begin{array}{l}\text { Primary carcinoma (mass) } \\
\text { RUL and ML, satellite } \\
\text { nodules RLL }\end{array}$ & $\begin{array}{l}\text { Cisplatin/ } \\
\text { vinorelbine, } \\
\text { then } \\
\text { carboplatin/ } \\
\text { pemetrexed }\end{array}$ & Yes & $\begin{array}{l}\text { RUL+ML lobectomy bilateral } \\
\text { metastatic adenocarcinoma }\end{array}$ & Oct 2017 & No & $\begin{array}{l}\text { RUL +ML lobectomy bilateral } \\
\text { metastatic adenocarcinoma }\end{array}$ \\
\hline
\end{tabular}




\begin{tabular}{|c|c|c|c|c|c|c|c|c|c|c|c|}
\hline $\begin{array}{l}\text { Case } \\
\text { number }\end{array}$ & $\begin{array}{l}\text { Time of } \\
\text { onset of } \\
\text { ICI }\end{array}$ & $\begin{array}{l}\text { ICI agent } \\
\text { (clinical } \\
\text { trial or RC) }\end{array}$ & $\begin{array}{l}\text { Events between onset of } \\
\mathrm{ICI} \text { and IP }\end{array}$ & $\begin{array}{l}\text { Time of IP } \\
\text { onset }\end{array}$ & $\begin{array}{l}\text { Time of IP } \\
\text { onset after } \\
\text { beginning of } \\
\text { ICI months }\end{array}$ & $\begin{array}{l}\text { IP severity grade } \\
\text { according to } \\
\text { CTCAE } 4.0\end{array}$ & Symptoms & $\begin{array}{l}\text { Diagnostic } \\
\text { method }\end{array}$ & $\begin{array}{l}\text { ICI interruption or } \\
\text { discontinuation }\end{array}$ & Steroid treatment & IP outcome \\
\hline 1 & April 2015 & $P(R C)$ & $\begin{array}{c}\text { Concurrent Pseudomonas } \\
\text { aeruginosa pneumonia }\end{array}$ & June 2017 & 26 & 4 & $\begin{array}{l}\text { Respiratory } \\
\text { failure }\end{array}$ & BAL & $\begin{array}{l}\text { Discontinued June } \\
2017\end{array}$ & Yes & $\begin{array}{l}\text { Death after initial clinical } \\
\text { and radiological } \\
\text { improvement }\end{array}$ \\
\hline 2 & Aug 2016 & $\begin{array}{l}N \text { (clinical } \\
\text { trial) }\end{array}$ & $\begin{array}{l}\text { Cryotherapy of the chest } \\
\text { wall metastasis }\end{array}$ & Oct 2017 & 13 & 3 & Dyspnoea & $\begin{array}{l}\text { Response to } \\
\text { therapy }\end{array}$ & $\begin{array}{l}\text { Discontinued Sept } \\
\quad 2017\end{array}$ & Yes & $\begin{array}{l}\text { Clinical and radiological } \\
\text { improvement }\end{array}$ \\
\hline 3 & July 2016 & $\begin{array}{l}\mathrm{I}+\mathrm{N} \text { then } \mathrm{N} \\
\quad(\mathrm{RC})\end{array}$ & No & April 2017 & 9 & 1 & None & $\begin{array}{l}\text { Histology } \\
\text { (surgical } \\
\text { excision) }\end{array}$ & No & No & $\begin{array}{l}\text { Clinical and radiological } \\
\text { improvement }\end{array}$ \\
\hline 4 & Dec 2016 & $\begin{array}{l}\mathrm{I}+\mathrm{N} \text { then } \mathrm{N} \\
(\mathrm{RC})\end{array}$ & No & $\begin{array}{l}\text { March } \\
2017\end{array}$ & 3 & 3 & $\begin{array}{l}\text { Dyspnoea, } \\
\text { cough }\end{array}$ & BAL & $\begin{array}{l}\text { Discontinued } \\
\text { March } 2017\end{array}$ & Yes & $\begin{array}{l}\text { Clinical and radiological } \\
\text { improvement }\end{array}$ \\
\hline 5 & July 2016 & $\begin{array}{l}\mathrm{I}+\mathrm{N} \text { then } \mathrm{N} \\
(\mathrm{RC})\end{array}$ & No & Dec 2016 & 5 & 1 & None & BAL & $\begin{array}{l}\text { Discontinued Dec } \\
2016\end{array}$ & No & $\begin{array}{l}\text { Clinical and radiological } \\
\text { improvement }\end{array}$ \\
\hline 6 & Nov 2016 & $N(R C)$ & No & Feb 2017 & 2 & 3 & Dyspnoea & $\begin{array}{l}\text { Response to } \\
\text { therapy }\end{array}$ & $\begin{array}{l}\text { Discontinued Feb } \\
2017\end{array}$ & Yes & $\begin{array}{l}\text { Clinical and radiological } \\
\text { improvement }\end{array}$ \\
\hline 7 & April 2016 & $\begin{array}{l}\mathrm{D}+\mathrm{T} \text { (clinical } \\
\text { trial) }\end{array}$ & No & June 2016 & 2 & 3 & $\begin{array}{l}\text { Dyspnoea, } \\
\text { cough }\end{array}$ & BAL & $\begin{array}{l}\text { Discontinued June } \\
2016\end{array}$ & Yes & $\begin{array}{l}\text { Clinical and radiological } \\
\text { improvement }\end{array}$ \\
\hline 8 & June 2016 & $N(R C)$ & No & July 2017 & 13 & 1 & None & BAL & $\begin{array}{l}\text { Discontinued June } \\
2017\end{array}$ & Yes & $\begin{array}{l}\text { Clinical and radiological } \\
\text { improvement }\end{array}$ \\
\hline 9 & May 2015 & I (clinical trial) & No & Sept 2015 & 4 & 1 & None & $\begin{array}{l}\text { BAL and } \\
\text { histology }\end{array}$ & $\begin{array}{l}\text { Discontinued June } \\
2017\end{array}$ & Yes & $\begin{array}{l}\text { Clinical and radiological } \\
\text { improvement }\end{array}$ \\
\hline 10 & Oct 2015 & $N(R C)$ & No & July 2016 & 8 & 4 & Dyspnoea & BAL & $\begin{array}{l}\text { Discontinued July } \\
2016\end{array}$ & Yes & $\begin{array}{l}\text { Clinical and radiological } \\
\text { improvement }\end{array}$ \\
\hline 11 & Aug 2016 & $\mathrm{I}+\mathrm{N}(\mathrm{RC})$ & No & Oct 2016 & 2 & 1 & None & Histology & $\begin{array}{l}\text { Discontinued Oct } \\
2016\end{array}$ & Yes & $\begin{array}{l}\text { Clinical and radiological } \\
\text { improvement }\end{array}$ \\
\hline 12 & $\begin{array}{l}\text { March } \\
2017\end{array}$ & $\mathrm{P}$ (clinical trial) & & Sept 2017 & 6 & 1 & $\begin{array}{l}\text { Dyspnoea, } \\
\text { cough }\end{array}$ & BAL & No & No & Slowed progression \\
\hline 13 & Sept 2016 & $N(R C)$ & $\begin{array}{l}\text { Unproven superimposed } \\
\text { infection of the RUL }\end{array}$ & Feb 2017 & 5 & 3 & Dyspnoea & BAL & $\begin{array}{l}\text { Discontinued Feb } \\
2017\end{array}$ & Yes & $\begin{array}{l}\text { Clinical and radiological } \\
\text { improvement }\end{array}$ \\
\hline 14 & Sept 2010 & I (RC) & No & Oct 2010 & 1 & 3 & Cough & BAL & $\begin{array}{l}\text { Discontinued Oct } \\
2010\end{array}$ & Yes & $\begin{array}{l}\text { Clinical and radiological } \\
\text { improvement }\end{array}$ \\
\hline 15 & Aug 2016 & $N(R C)$ & RT & Feb 2018 & 17 & 3 & Dyspnoea & BAL & $\begin{array}{l}\text { Discontinued Jan } \\
2018\end{array}$ & Yes & $\begin{array}{l}\text { Clinical and radiological } \\
\text { improvement }\end{array}$ \\
\hline
\end{tabular}

ICI: immune checkpoint inhibitors; RC: routine care; IP: immune-related pneumonitis; CTCAE: Common Terminology Criteria for Adverse Events; P: pembrolizumab; N: nivolumab;

I: ipilimumab; D: durvalumab; T: tremelimumab; RUL: right upper lobe; RT: radiotherapy; BAL: bronchoalveolar lavage. 
TABLE 3 Details of thoracic radiotherapy (RT)

\begin{tabular}{|c|c|c|c|c|c|c|}
\hline $\begin{array}{l}\text { Case } \\
\text { number }\end{array}$ & Type & Site & Dose Gy & Fractions & $\begin{array}{l}\text { Radiation } \\
\text { pneumonitis }\end{array}$ & $\begin{array}{l}\text { Time between RT and } \\
\text { immunotherapy } \\
\text { months }\end{array}$ \\
\hline
\end{tabular}

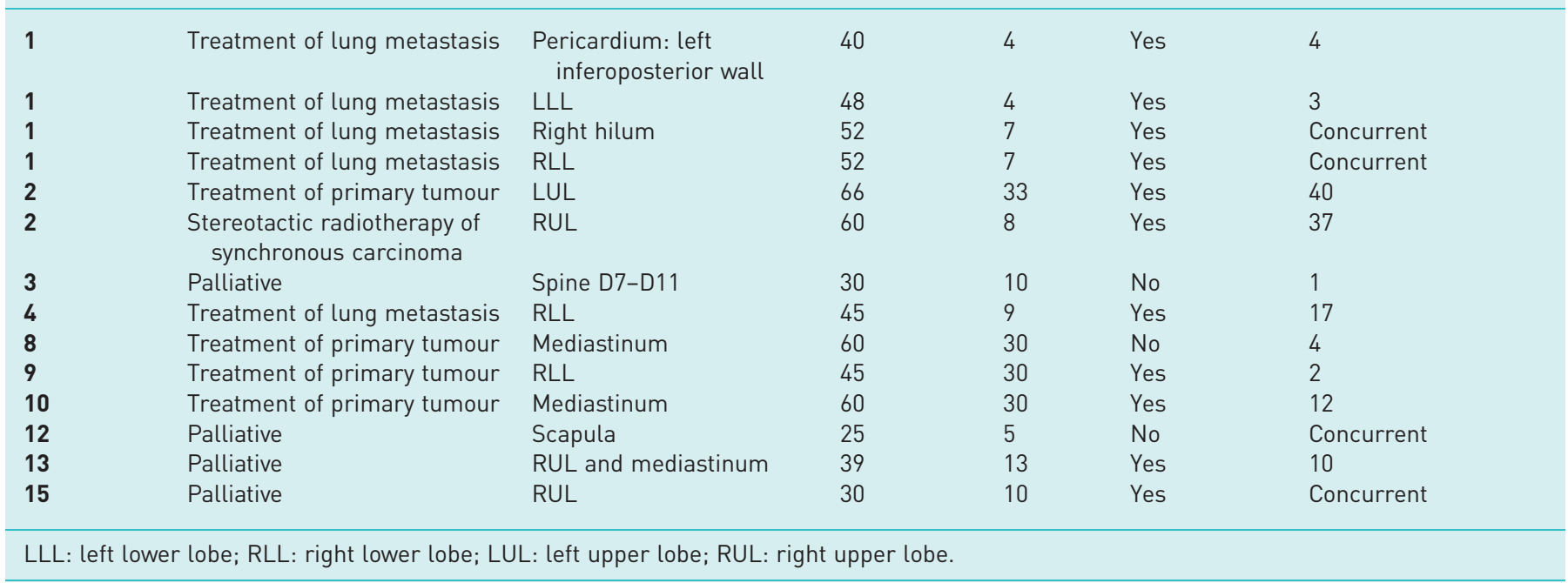

baseline scan. Imaging findings compatible with radiation-induced pneumonitis occurring within the field of RT and within 6 months after treatment were observed in six cases. These radiation-induced abnormalities were not considered for the specific description of the IP pattern due to potential overlapping features of radiation pneumonitis (RP) and IP.

\section{TABLE 4 Computed tomography (CT) analysis and comparison with bronchoalveolar lavage (BAL) results}

\begin{tabular}{|c|c|c|c|c|c|c|}
\hline $\begin{array}{l}\text { Case } \\
\text { number }\end{array}$ & $\begin{array}{l}\text { Lung regions } \\
\text { involved by IP }\end{array}$ & Additional findings & RT & $\begin{array}{l}\text { Radiation } \\
\text { pneumonitis }\end{array}$ & CT pattern & $\begin{array}{l}\text { BAL/biopsy } \\
\text { pattern }\end{array}$ \\
\hline 1 & Diffuse & $\begin{array}{l}\text { Pleural effusion, increasing GGOs } \\
\text { over time }\end{array}$ & Yes & Yes & DAD and OP & Neutrophilic \\
\hline 2 & UR, LR, ML, LL & & Yes & Yes & $\mathrm{OP}$ & NA \\
\hline 3 & UR & Lung nodule increasing over time & Yes & No & Nodule & $\begin{array}{r}\text { Lymphocytic } \\
\text { (histology) }\end{array}$ \\
\hline 6 & Diffuse & $\begin{array}{l}\text { Pre-existing lobular attenuation, } \\
\text { excluding HP }\end{array}$ & No & NA & cNSIP and OP & NA \\
\hline 7 & Diffuse & $\begin{array}{l}\text { Migratory pattern, pleural } \\
\text { effusion }\end{array}$ & No & NA & $\begin{array}{l}\text { Mixed OP and cNSIP } \pm \\
\text { other unspecified ILD }\end{array}$ & Lymphocytic \\
\hline 8 & UR, ML, LL & Migratory pattern & Yes & No & $\mathrm{OP}$ & Lymphocytic \\
\hline 9 & MR, LR & Migratory pattern & Yes & Yes & $\mathrm{OP}$ & Mixed \\
\hline 14 & Diffuse & $\begin{array}{l}\text { Perilymphatic micronodularity, } \\
\text { peribronchovascular } \\
\text { consolidation and mosaic } \\
\text { perfusion pattern }\end{array}$ & No & NA & LIP, cNSIP, DAD & Lymphocytic \\
\hline 15 & Diffuse & $\begin{array}{l}\text { Migratory pattern and pleural } \\
\text { effusion }\end{array}$ & Yes & No & $\mathrm{OP}$ & Mixed \\
\hline
\end{tabular}

IP: immune-related pneumonitis; RT: radiation therapy; BAL: bronchoalveolar lavage; UR: upper right; LR: lower right; ML: medium left; LL: lower left; MR: medium right; UL: upper left; GGO: ground-glass opacity; HP: hypersensitivity pneumonitis; NA: not available; DAD: diffuse alveolar damage; OP: organising pneumonia; cNSIP: cellular nonspecific interstitial pneumonia; LIP: lymphoid interstitial pneumonia; ILD: interstitial lung disease. 
TABLE 5 Correlation between immune-related pneumonitis (IP) extension and underlying lung conditions

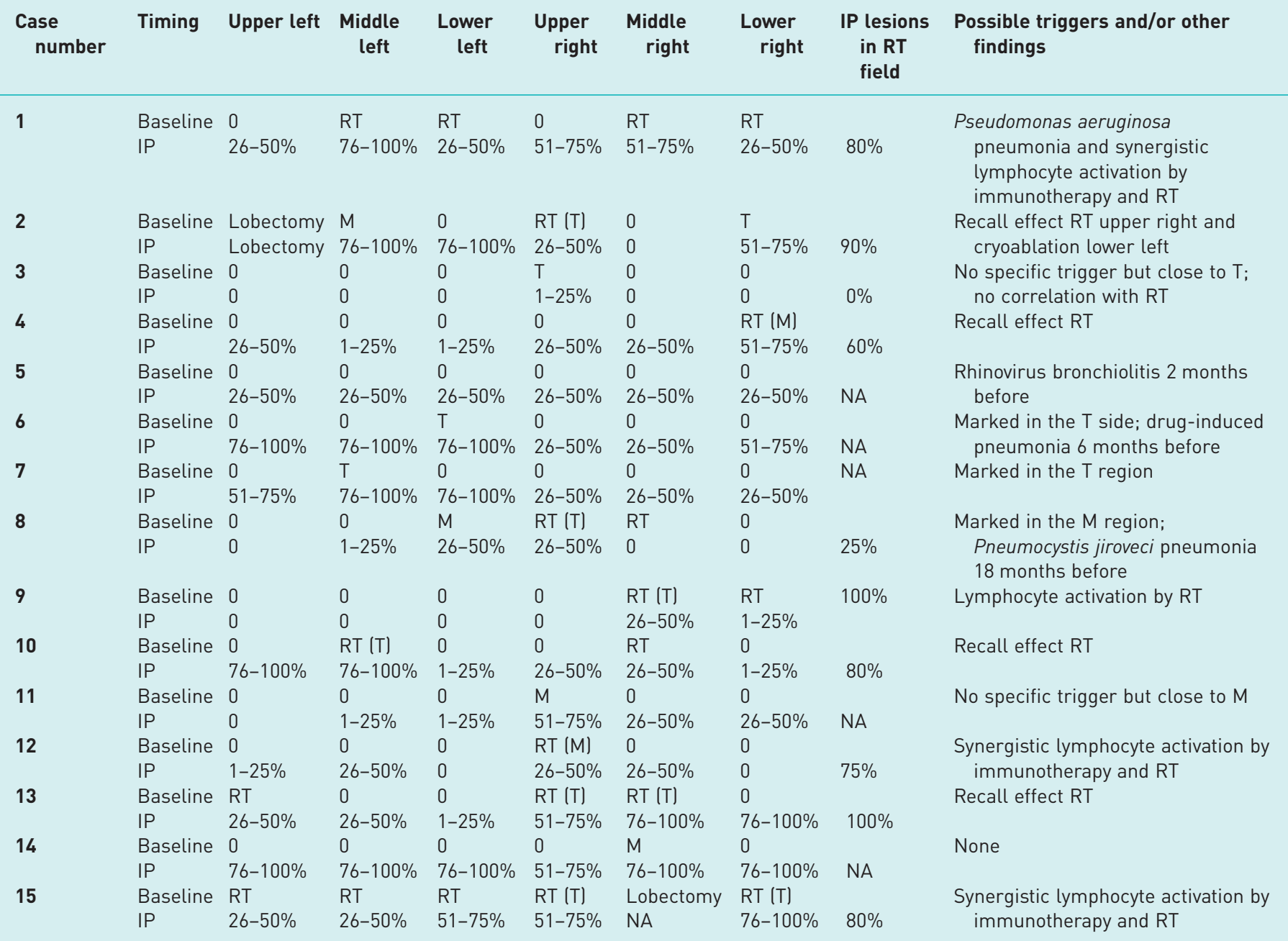

In patients who underwent radiation therapy, radiation therapy (RT) is used in the baseline row to identify the lung regions included in the radiation field. The tumour manifestation (primary tumour (T) or metastasis (M)) is added in parenthesis whenever the tumour manifestation was located in that irradiated region. NA: not available.

Two lung regions were missing on the IP scan due to surgery; therefore, 88 regions were evaluated. The IP scan showed IP-related lesions in $72(81 \%)$ out of 88 pulmonary regions, whereas $16(19 \%)$ out of 88 regions were free of IP. The IP-free regions were also free of tumour, metastasis or RT on the baseline scan. Conversely, a significant prevalence of IP-related lesions was found in the regions of primary tumour $(n=4)$, metastasis $(n=4)$ and RT $(n=21)(p=0.009)$, with an odds ratio of 10.8 for IP occurrence in these regions $(\mathrm{p}=0.024)$. The analysis of $\mathrm{RT}$ cases alone showed also a trend for a higher prevalence of IP in RT regions $(95 \%)$ compared to RT-naïve regions $(5 \%, \mathrm{p}=0.061)$.

Various imaging patterns, distributions and degrees of lung involvement were observed in the same patient and between patients (table 4). Nine patients (60\%) had IP-related abnormalities over all lung fields bilaterally. Regarding the overall pattern of interstitial lung disease, CT findings were consistent with pure OP in 7 patients $(47 \%)$, combined OP and NSIP in four cases $(27 \%)$, and combined OP and DAD in one patient $(6 \%$; case 1$)$. In two cases, no characteristic features were detected and the pattern was considered undefined (12.5\%). A solitary nodule increasing in size over time was seen in one patient and led to surgical resection with pathological proof of IP (6\%). Pleural effusion was seen in three patients $(20 \%)$. A pure OP pattern was found more frequently in patients who underwent RT than those who did not $(70 \%$ versus $0 \%, \mathrm{p}=0.024)$, whereas NSIP features were identified more frequently in those who did not receive RT (100 versus $10 \%, \mathrm{p}=0.002$ ). Two illustrative cases are shown in figures 1 and 2 . 

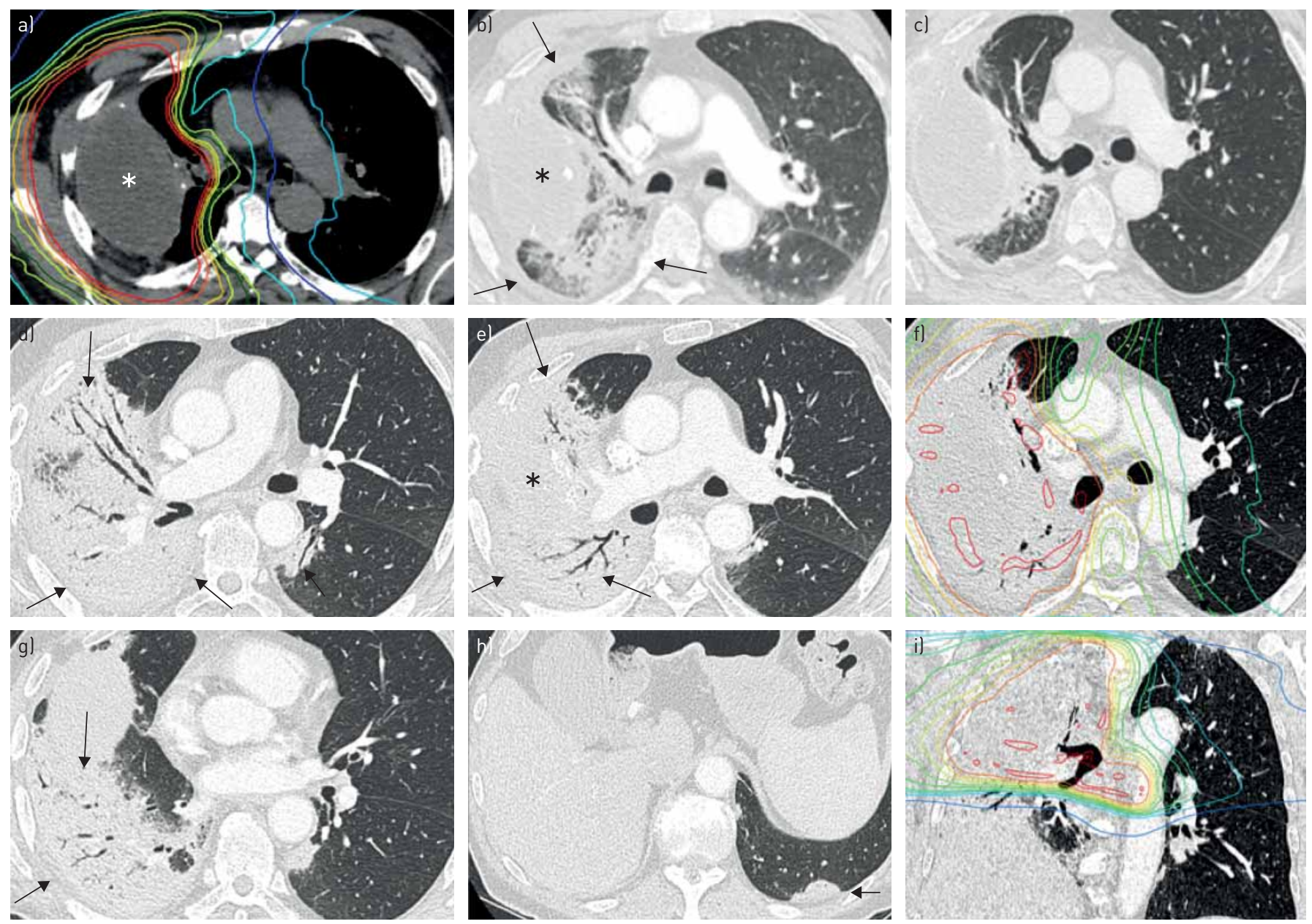

FIGURE 1 Immune-related pneumonitis grade 3 with organising pneumonia computed tomography (CT) pattern developed during nivolumab treatment in an 88-year-old man with non-small cell lung cancer infiltrating the third and fourth right ribs (cT3 cN1 cM0, stage IIIA). a) Radiation therapy field (*), $39 \mathrm{~Gy}$ in 13 fractions of $3 \mathrm{~Gy}$ (red: $50 \mathrm{~Gy}$; blue: $5 \mathrm{~Gy}$ ). b) CT scan performed 2 months after radiation therapy showing radiation-induced pneumonitis (arrows). Abnormalities in the contralateral lung are mainly due to poor inspiration. c) CT scan before starting nivolumab, 6 months after radiotherapy, showing almost complete resolution of the radiation-induced pneumonitis. After 4 months of nivolumab, the patient developed grade 3 dyspnoea. $d$ and e) New alveolar and partly peribronchovascular consolidations (arrows) are visible. f) The consolidations are located within the radiation field, as revealed by the fusion CT-radiation therapy planning image (red: 50 Gy; blue: 5 Gy). However, g-i) some consolidations are also located outside the radiation field in the fusion CT-radiation therapy planning image (i, arrows), especially at the right lower lobe (g) and in a subpleural location on the contralateral side (h, arrow).

\section{Discussion}

In the present study, we found that IP triggered by ICIs preferentially locates within the regions of primary cancer, lung metastasis and/or RT fields ( $\mathrm{p}=0.009)$. Even if IP could involve adjacent or distant areas, including the contralateral lung, the probability that IP abnormalities affected primary tumour, metastasis and/or RT regions was 10.8 times higher than in the others. While no increased risk of IP has been demonstrated so far when using combined RT-ICIs [2, 3, 19], this study is the first to describe IP abnormalities predominantly within the irradiated areas, as well as within the lung regions involved by the cancer, either the primary tumour or metastasis in RT-naïve patients.

Although the pathogenesis of IP is largely unknown, immune dysregulation likely plays a role, as pathological specimens are typically characterised by lymphocyte infiltration [20]. It can be hypothesised that during immunotherapy, lung homeostasis is altered and an autoimmune reaction is triggered [21, 22]. In this setting, it is conceivable that the predominant reaction is seen firstly where the lymphocytes are pooled, such as around tumoral lesions or within the RT field, and it could then diffuse to the surrounding areas and, in some cases, to distant regions. Moreover, in addition to RT, several pulmonary events, including pulmonary infection, cryoablation of a lung metastasis and chemotherapy-induced pneumonia, could have further altered immune homeostasis (table 5). These local conditions could have promoted inflammatory responses and caused a hyperactivation of the immune system leading to irAEs. 

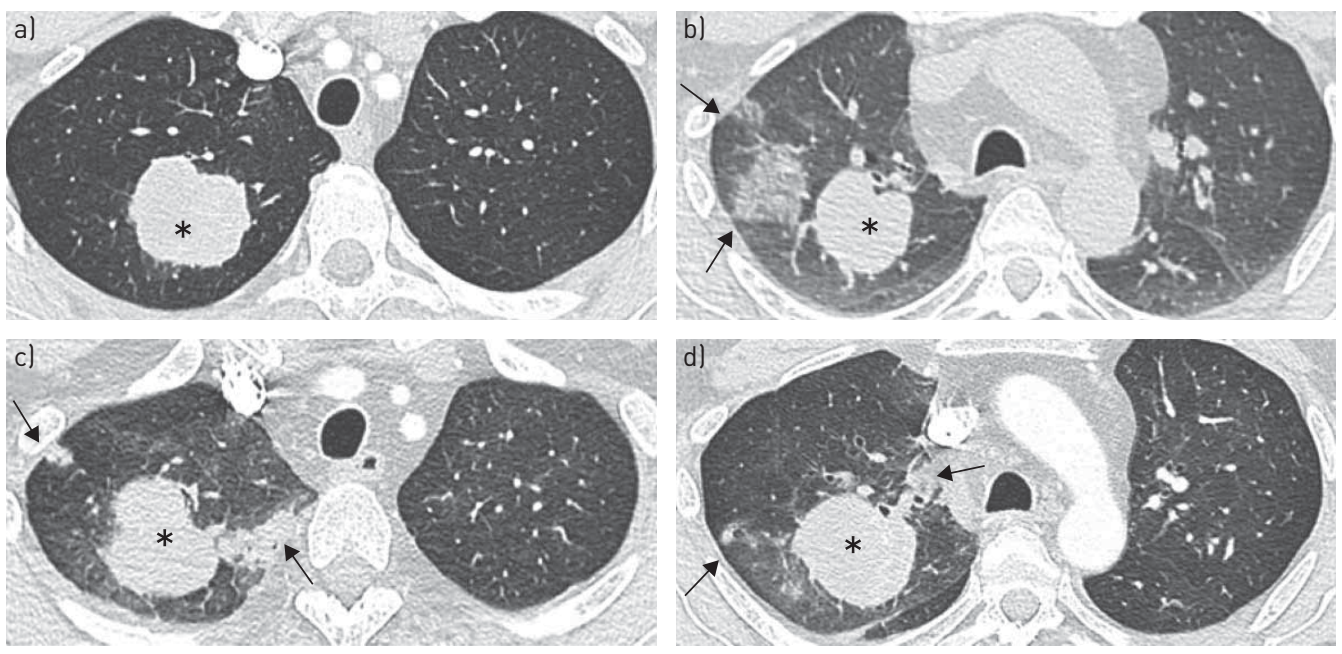

FIGURE 2 Immune-related pneumonitis grade 1 with organising pneumonia features developed after two cycles of combined therapy with nivolumab and ipilimumab in a 46-year-old man with metastatic melanoma. a) Lung metastasis $(*)$ of the right upper lobe on baseline computed tomography (CT) scan. b) The restaging CT performed after the first cycle shows a focal area of ground-glass opacity (arrows) in the vicinity of the lung metastasis $(*)$. The patient was asymptomatic. $c$ and d) 1 month later, the ground-glass opacity has disappeared but new-onset subpleural and peribronchovascular consolidations (arrows) are seen close to the metastasis. No CT abnormalities are found in the contralateral lung.

Our finding of a preferential location of IP to RT fields is of particular interest. Several trials have raised the interest of administering immunotherapy with other treatments, particularly RT, since their antitumoural effects may be enhanced when combined [2, 6-9, 23].

In addition to its antitumoural effects, RT promotes an immune, mainly T-cell-mediated, response known as "in situ vaccination", which plays an important role in the outcome of RT [21, 22, 24]. This immune system activation is not restricted to the RT field but also induces a systemic, tumour-specific immune response outside the RT field due to immunogenic cell death. This phenomenon could be related to the "abscopal effect", a radiation-induced mechanism where reduction of tumour burden is observed not only in the RT field but also outside. The interest of the abscopal effect has been recently underlined by pre-clinical and clinical evidence of tumour shrinking outside the radiation field when RT was used in combination with ICIs $[22,24,25]$. In case 15 , treated by combined RT-ICIs, a significant response of all metastases was observed after the irradiation of the largest lesion, while no tumour reduction was previously observed during immunotherapy alone. Interestingly, a predominant location of IP was seen around all metastases.

Beside the hopeful synergic antitumoural effects of combined ICI-RT, particular attention has been paid to irAEs, including lung toxicities in case of thoracic RT, as it could be hypothesised that these toxicities would be increased by the enhanced pro-inflammatory activities. However, the first clinical data suggest that a safe and tolerable profile is maintained $[2,6,23,26]$. Nevertheless, it has been recently described that immunotherapy may cause an inflammatory reaction in previously irradiated area as a RP or even years after irradiation, owing to the known "recall effect" [5, 14-16, 27-29]. These biological effects reinforce the interest of combined radiotherapy and immunotherapy, and could partly explain the predominant location of IP abnormalities within RT fields [2-4, 19]. Although in case of previous RT, it may be difficult to strictly attribute observed changes to either IP or RP, our observations in RT-free cases rules out a recall effect alone. Further studies are needed to investigate whether the interval between RT and immunotherapy administration may influence the incidence of pneumonitis within the radiation field. Similarly to RT, cryoablation causes direct neoplastic cell death and stimulates a local immune response so that, when combined with immunotherapy, it may also act by immunogenic cell death [30].

Similarly to previous studies, the detailed evaluation of CT characteristics did not show a specific pattern of IP $[12,13,31]$. Although OP and NSIP features were frequently observed, a specific pattern could not be defined in some cases, as previously reported by NAIDOo et al. [12]. In patient 1, an increasing ground-glass opacification pattern on a background of subpleural consolidations was detected on consecutive scans, suggesting an $\mathrm{OP}$ evolving to DAD, which matched the worsening of the clinical condition towards respiratory failure. Such an "evolving pattern" has rarely been reported [12]. Interestingly, in the present study, a pure OP pattern was more frequently found in patients who 
underwent RT ( $\mathrm{p}=0.024)$, whereas NSIP features were more commonly seen in patients who did not have RT $(\mathrm{p}=0.002)$.

The present study has several limitations, including its retrospective design, the small sample size, and variations in tumour histology and treatments. Additional studies with larger sample size are required to confirm our results. A correlation between ICI efficacy on the tumoural burden and occurrence of IP was not analysed, and also requires further studies. One could argue that confusion may have occurred between radiation-induced pneumonia and IP. However, among the 10 cases who underwent RT, eight had opacities attributable to IP outside the radiation field, making the diagnosis of pure RP unlikely. In the two remaining cases ( 9 and 13), 100\% of opacities attributed to IP were located in the radiation field (table 5) but in both of them, IP occurred $\geqslant 6$ months after RT, again making the diagnosis of pure RP unlikely. Additionally, similar morphological abnormalities were also observed in RT-naïve patients, especially in lung areas involved by tumour (either primary tumour or metastasis), which also argues against pure RP. We therefore believe that no confusion occurred between RP and IP in the present study. Nevertheless, further studies are required to examine the relationships between these two events, as well as the possible triggering role of other events such as lung infections, which might also alter lung homeostasis and play a role in IP development.

In conclusion, the present study showed a predominance of IP features in the site of cancer and/or RT areas. Although the mechanisms remain unclear, this finding could be related to a higher pool of lymphocytes in these areas during ICI treatment. This immune reaction could then extend to adjacent regions as well as distant ones by cascade activation. A comprehensive understanding of IP pathogenesis, causes and imaging features may allow earlier detection, with a relevant impact on patient care. In patients receiving ICIs, the detection of new-onset radiological abnormalities within either the region of neoplastic lesion or the RT field should alert the clinician to consider IP in the differential diagnosis, allowing prompt and correct management.

Conflict of interest: C. Pozzessere has nothing to disclose. H. Bouchaab has nothing to disclose. R. Jumeau has nothing to disclose. I. Letovanec has nothing to disclose. C. Daccord has nothing to disclose. J. Bourhis has nothing to interest. J.O. Prior has nothing to disclose. S. Peters reports personal fees for advisory boards and honoraria from Abbvie, Amgen, AstraZeneca, Bayer, Biocartis, Boehringer Ingelheim, Bristol-Myers Squibb, Clovis, Daiichi Sankyo, Debiopharm, Eli Lilly, F. Hoffmann-La Roche, Foundation Medicine, Illumina, Janssen, Merck Sharp and Dohme, Merck Serono, Merrimack, Novartis, Pharma Mar, Pfizer, Regeneron, Sanofi, Seattle Genetics and Takeda; personal fees for talks and honoraria from AstraZeneca, Boehringer Ingelheim, Bristol-Myers Squibb, Eli Lilly, F. Hoffmann-La Roche, Merck Sharp and Dohme, Novartis, Pfizer and Takeda; non-financial support for investigation in trials sponsored by Amgen, AstraZeneca, Boehringer Ingelheim, Bristol-Meyers Squibb, Clovis, F. Hoffmann-La Roche, Illumina, Merck Sharp and Dohme, Merck Serono, Novartis and Pfizer; non-financial support for a talk and an honorarium from Sanofi, all outside the submitted work. R. Lazor reports personal fees for travel costs for continuing education from Boehringer Ingelheim, Roche and Vifor, outside the submitted work. C. Beigelman-Aubry reports personal fees for lectures from Gilead, AstraZeneca and Boehringer, outside the submitted work.

\section{References}

Mellman I, Coukos G, Dranoff G. Cancer immunotherapy comes of age. Nature 2011; 480: 480-489.

2 Antonia SJ, Villegas A, Daniel D, et al. Durvalumab after chemoradiotherapy in stage III non-small cell lung cancer. N Engl J Med 2017; 377: 1919-1929.

3 Shaverdian N, Lisberg AE, Bornazyan $\mathrm{K}$, et al. Previous radiotherapy and the clinical activity and toxicity of pembrolizumab in the treatment of non-small-cell lung cancer: a secondary analysis of the KEYNOTE-001 phase 1 trial. Lancet Oncol 2017; 18: 895-903.

4 Voong KR, Naidoo J, Ettinger DS. The next frontier in non-small cell lung cancer: synergizing radiation therapy and immune checkpoint blockade. Clin Adv Hematol Oncol 2017; 15: 615-625.

5 Li M, Gan L, Song A, et al. Rethinking pulmonary toxicity in advanced non-small cell lung cancer in the era of combining anti-PD-1/PD-L1 therapy with thoracic radiotherapy. Biochim Biophys Acta Rev Cancer 2019; 1871: 323-330.

6 Peters S, Felip E, Dafni U, et al. Safety evaluation of nivolumab added concurrently to radiotherapy in a standard first line chemo-radiotherapy regimen in stage III non-small cell lung cancer - the ETOP NICOLAS trial. Lung Cancer 2019; 133: 83-87.

7 Bauml JM, Mick R, Ciunci C, et al. Pembrolizumab after completion of locally ablative therapy for oligometastatic non-small cell lung cancer: a phase 2 trial. JAMA Oncol 2019; 5: 1283-1290.

8 Walker J, Loo BW, Jr. Radiotherapy and immunotherapy - shining further together. JAMA Oncol 2019; 5: 1291-1292.

9 Theelen W, Peulen HMU, Lalezari F, et al. Effect of pembrolizumab after stereotactic body radiotherapy vs pembrolizumab alone on tumor response in patients with advanced non-small cell lung cancer: results of the PEMBRO-RT phase 2 randomized clinical trial. JAMA Oncol 2019; 5: 1276-1282.

10 Haanen J, Carbonnel F, Robert C, et al. Management of toxicities from immunotherapy: ESMO clinical practice guidelines for diagnosis, treatment and follow-up. Ann Oncol 2017; 28: iv119-iv142.

11 Khunger M, Rakshit S, Pasupuleti V, et al. Incidence of pneumonitis with use of programmed death 1 and programmed death-ligand 1 inhibitors in non-small cell lung cancer: a systematic review and meta-analysis of trials. Chest 2017; 152: 271-281. 
12 Naidoo J, Wang X, Woo KM, et al. Pneumonitis in patients treated with anti-programmed death-1/programmed death ligand 1 therapy. J Clin Oncol 2017; 35: 709-717.

13 Nishino M, Ramaiya NH, Awad MM, et al. PD-1 inhibitor-related pneumonitis in advanced cancer patients: radiographic patterns and clinical course. Clin Cancer Res 2016; 22: 6051-6060.

14 Louvel G, Bahleda R, Ammari S, et al. Immunotherapy and pulmonary toxicities: can concomitant immune-checkpoint inhibitors with radiotherapy increase the risk of radiation pneumonitis? Eur Respir J 2018; 51: 1701737.

15 Shibaki R, Akamatsu H, Fujimoto M, et al. Nivolumab induced radiation recall pneumonitis after two years of radiotherapy. Ann Oncol 2017; 28: 1404-1405.

16 Tamiya A, Tamiya M, Nakahama K, et al. Correlation of radiation pneumonitis history before nivolumab with onset of interstitial lung disease and progression-free survival of patients with pre-treated advanced non-small cell lung cancer. Anticancer Res 2017; 37: 5199-5205.

17 Hansell DM, Bankier AA, MacMahon H, et al. Fleischner Society: glossary of terms for thoracic imaging. Radiology 2008; 246: 697-722.

18 Travis WD, Costabel U, Hansell DM, et al. An official American Thoracic Society/European Respiratory Society statement: Update of the international multidisciplinary classification of the idiopathic interstitial pneumonias. Am J Respir Crit Care Med 2013; 188: 733-748.

19 Peters S, Ruysscher DD, Dafni U, et al. Safety evaluation of nivolumab added concurrently to radiotherapy in a standard first line chemo-RT regimen in unresectable locally advanced NSCLC: the ETOP NICOLAS phase II trial. J Clin Oncol 2018; 36: 8510-8510.

20 Postow MA, Sidlow R, Hellmann MD. Immune-related adverse events associated with immune checkpoint blockade. N Engl J Med 2018; 378: 158-168.

21 Lhuillier C, Vanpouille-Box C, Galluzzi L, et al. Emerging biomarkers for the combination of radiotherapy and immune checkpoint blockers. Semin Cancer Biol 2018; 52: 125-134.

22 Vanpouille-Box C, Formenti SC, Demaria S. Toward precision radiotherapy for use with immune checkpoint blockers. Clin Cancer Res 2018; 24: 259-265.

23 Verma V, Cushman TR, Selek U, et al. Safety of combined immunotherapy and thoracic radiation therapy: analysis of 3 single-institutional phase I/II trials. Int J Radiat Oncol Biol Phys 2018; 101: 1141-1148.

24 Herrera FG, Bourhis J, Coukos G. Radiotherapy combination opportunities leveraging immunity for the next oncology practice. CA Cancer J Clin 2017; 67: 65-85.

25 Liu Y, Dong Y, Kong L, et al. Abscopal effect of radiotherapy combined with immune checkpoint inhibitors. J Hematol Oncol 2018; 11: 104.

26 Miyamoto S, Nomura R, Sato K, et al. Nivolumab and stereotactic radiation therapy for the treatment of patients with stage IV non-small cell lung cancer. Jpn J Clin Oncol 2019; 49: 160-164.

27 Burris HA 3rd, Hurtig J. Radiation recall with anticancer agents. Oncologist 2010; 15: 1227-1237.

28 Wirsdorfer F, de Leve S, Jendrossek V. Combining radiotherapy and immunotherapy in lung cancer: can we expect limitations due to altered normal tissue toxicity? Int J Mol Sci 2018; 20: 24

29 Bang A, Schoenfeld JD. Immunotherapy and radiotherapy for metastatic cancers. Ann Palliat Med 2019; 8 : 312-325.

30 Abdo J, Cornell DL, Mittal SK, et al. Immunotherapy plus cryotherapy: potential augmented abscopal effect for advanced cancers. Front Oncol 2018; 8: 85.

31 Kato T, Masuda N, Nakanishi Y, et al. Nivolumab-induced interstitial lung disease analysis of two phase II studies patients with recurrent or advanced non-small-cell lung cancer. Lung Cancer 2017; 104: 111-118. 